\title{
Randomized, Double-Blind, and Placebo-Controlled Clinic Report of Intranasal Low-Intensity Laser Therapy on Vascular Diseases
}

\author{
Timon Cheng-Yi Liu, ${ }^{1,2}$ Lei Cheng, ${ }^{1}$ Wen-Juan Su, ${ }^{3}$ Yi-Wen Zhang, ${ }^{3}$ \\ Yun Shi, ${ }^{3}$ Ai-Hong Liu, ${ }^{3}$ Li-Li Zhang, ${ }^{3}$ and Zhuo-Ya Qian ${ }^{3}$ \\ ${ }^{1}$ Laboratory of Laser Sports Medicine, South China Normal University, Guangzhou 510006, China \\ ${ }^{2}$ Ministry of Education of China Key Laboratory of Laser Life Science, South China Normal University, \\ Guangzhou 510006, China \\ ${ }^{3}$ Medicine Department, Shanghai Pudong District Cancer Prevention and Cure Hospital, Pudong District, \\ Shanghai 200126, China \\ Correspondence should be addressed to Timon Cheng-Yi Liu, liutcy@scnu.edu.cn
}

Received 27 January 2012; Revised 21 March 2012; Accepted 27 March 2012

Academic Editor: Rui Duan

Copyright () 2012 Timon Cheng-Yi Liu et al. This is an open access article distributed under the Creative Commons Attribution License, which permits unrestricted use, distribution, and reproduction in any medium, provided the original work is properly cited.

\begin{abstract}
The intranasal low intensity GaInP/AlGaInP diode $650 \mathrm{~nm}$ laser therapy (ILGLT) might improve blood lipid and hemorheologic behavior of patients in view of its previous research, but it should be further supported by a randomized, double-blind, and placebo-controlled clinical study. In this paper, 90 patients with coronary heart disease or cerebral infarction were randomly divided into two groups, 60 in the treatment group and 30 in the control group, and were blindly treated with ILGLT at 8.38 and $0 \mathrm{~mW} / \mathrm{cm}^{2}$ for $30 \mathrm{~min}$ each time once a day ten days each session for two sessions between which there were three days for rest, respectively. Fasting blood lipid such as total cholesterol and low/high-density lipoprotein cholesterol and hemorheologic behavior such as blood viscosity, plasma viscosity, redox viscosity and red blood cell aggregation were assessed before the first treatment and after the two sessions and were found to be significantly improved by ILGLT. It was concluded that ILGLT may improve blood lipid and hemorheologic behavior of patients with coronary heart disease or cerebral infarction.
\end{abstract}

\section{Introduction}

Photobiomodulation (PBM) is a modulation of laser irradiation or monochromatic light (LI) on biosystems, which stimulates or inhibits biological functions but does not result in irreducible damage [1]. The LI used in PBM is always low-intensity LI (LIL), $\sim 10 \mathrm{~mW} / \mathrm{cm}^{2}$, which includes the LI used in the so-called ultralow level laser therapy [2]. From 1989 on, many Russian groups have studied the therapeutic effects of intranasal LIL on the local inflammation in vasomotor rhinitis $[3,4]$ and acute and chronic maxillary sinusitis [5]. In the mainland of China, intranasal LIL has been studied to treat internal diseases and the special treatment was called intranasal low intensity laser therapy (ILILT) since 1998 [6, 7]. ILILT has been applied to treat hyperlipidemia, the blood-stasis syndrome of coronary heart disease (CHD), myocardial infarction, and brain diseases such as insomnia, intractable headache, Alzheimer's disease, Parkinson's disease, poststroke depression, ache in head or face, migraine, cerebral thrombosis, diabetic peripheral neuropathy, cerebral infarction (CI), acute ischemic cerebrovascular disease, brain lesion, schizophrenia, cerebral palsy, and mild cognitive impairment $[6,7]$. The studies indicated that serum amyloid $\beta$ protein, malformation rate of erythrocytes, plasma cholecystokinin-octapeptide, the level of viscosity at lower shear rate $(3 / s)$, hematocrit $(\mathrm{HCT})$, and serum lipid decreased, respectively, and melatonin productionred cell deformability, superoxidase dismutase activity, and $\beta$ endorphin increased, respectively; blood circulation was improved, and immunity was regulated after ILILT $[6,7]$. 
ILILT might be a systemic effect. Nose-mediated therapeutics in traditional Chinese medicine (TCM) has been a very old system [8]. The nasal cavity has long been hypothesized to play an important role in climatic adaptation. The bony nasal cavity appears mostly associated with temperature, and the nasopharynx with humidity [9]. The nose might play more roles in disease treatment [69]. There are five possible pathways mediating the ILILT, olfactory nerve, nasal bone, blood cells, meridians in TCM and autonomic nervous system (ANS) $[6,7]$. As Wilson et al. have reviewed [10], some impaired olfaction in old age is associated with postmortem evidence of neurodegenerative disease, particularly neurofibrillary tangles and Lewy bodies. These associations, which are present even in the absence of dementia, may explain why olfactory impairment predicts important consequences of neurodegenerative conditions including cognitive decline, incidence of mild cognitive impairment and dementia, incidence of Parkinson's disease, worsening parkinsonian gait, and neuropsychiatric complications of Parkinson's disease [10]. Mesenchymal stem cells/marrow stromal cells (MSCs) in nasal bone might mediate ILILT. MSCs present a promising tool for its cell therapy and are currently being tested in US FDA-approved clinical trials for MI, stroke, meniscus injury, limb ischemia, graft-versus-host disease, autoimmune disorders, and so on. They have been extensively tested and proven effective in preclinical studies for these and many other disorders. It has been found that the therapeutic effects of bone marrow irradiation with LIL on rat MI have been more significant than the ones of MI irradiation, and the effects have been mediated by MSCs [11]. Blood cells mediate the therapeutic effects of intranasal PBM on the local inflammation. Tulebaev et al. have found the LIL-treated patients with vasomotor rhinitis showed a significant increase of $\mathrm{T}$ lymphocytes and a higher capacity of $\mathrm{T}$ cells to form the migration inhibition factor [3]. Shevrygin et al. have shown that LIL is effective in correction of microcirculatory disorders and tissue mechanisms of homeostasis in children with neurovegetative vasomotor rhinitis [4]. Kruchinina et al. have studied therapeutic effect of LIL on microcirculation of nasal mucosa in children with acute and chronic maxillary sinusitis, and found that laser therapy produced a positive effect on microcirculation and reduced the potential of relapses [5]. As it has been indicated in this paper, ILILT might improve blood cells. There are six meridians inside/around nose, stomach meridian of foot yang-ming, $d u$ meridian, yin-jiao meridian, yang-jiao meridian, large intestine meridian of hand yang-ming, and small intestine meridian of hand tai-yang. These six meridians can be irradiated by intranasal LIL through intranasal multiple reflex. They were supposed to mediate some of the therapeutic effects of ILILT. The central ANS functions at maintaining cardiovascular hemodynamics. There is crosstalk between nasal ANS and central ANS [12]. The central ANS activities significantly correlated with changes to the nasal airway during postural change. The central ANS, especially the sympathetic nervous system, may play a role in controlling nasal airway during postural change. Therefore, nasal ANS might mediate ILILT.

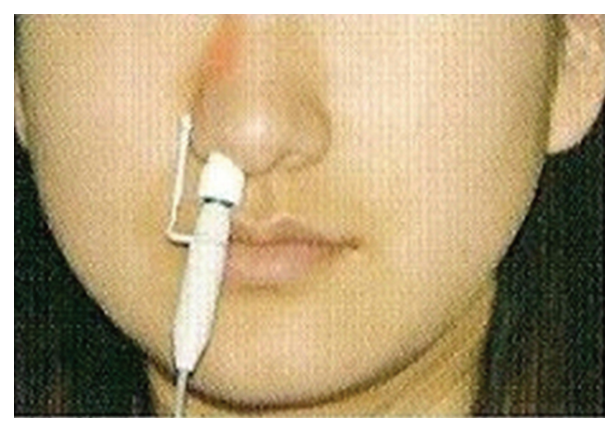

FIGURE 1: The setup of ILGLT at $650 \mathrm{~nm}$ and $8.38 \mathrm{~mW} / \mathrm{cm}^{2}$.

As it has been discussed above, ILILT might be a systemic effect. However, its studies have not been randomized, double-blind, and placebo-controlled. In this paper, a randomized, double-blind, and placebo-controlled clinical study was designed to assess the effects of intranasal low intensity GaInP/AlGaInP diode $650 \mathrm{~nm}$ laser therapy (ILGLT) on the blood lipid and hemorheologic behavior of patients with CHD or CI.

\section{Materials and Methods}

The study protocol was approved by the ethics committee of the Faculty of Medicine, Shanghai Pudong District Cancer Prevention and Cure Hospital, Shanghai, China. Informed consent was obtained from each participant prior to the start of treatment.

2.1. Patients. The 90 clinic patients, 49 men and 41 women, with CHD or CI in Shanghai Pudong District Cancer Prevention and Cure Hospital from January to March in 2008, were approached to participate in this study. By conventional WHO criteria, CHD was diagnosed in terms of electrocardiogram and coronary angiography, and CI was diagnosed in terms of coronal computer tomography appearance. The patients were divided randomly into treatment group with 60 patients and control group with 30 patients. There were no significant differences of gender, age, and type of disease between the two groups $(P>0.05)$ (Table 1).

2.2. Laser Irradiation. BLOODCARE Medical Laser Device manufactured with GaInP/AlGaInP diode $650 \mathrm{~nm}$ laser by Shanghai Taicheng (TCM) Technology and Development Co. Ltd. It has been applied to irradiate the patients intranasally (Figure 1) 30 min each time once a day ten days each session for two sessions between which there were 3 days for rest. The treatment group and the control group were irradiated at $8.38 \mathrm{~mW} / \mathrm{cm}^{2}$ (the spot area is $0.358 \mathrm{~cm}^{2}$ ) and $0 \mathrm{~mW} / \mathrm{cm}^{2}$, respectively, and no other treatments have been done.

2.3. Blood Viscosity Assays. There were no dietary restrictions on the patients, but the patients fasted before their blood was drawn. Blood samples were collected according to routine clinical protocol. Venous blood was withdrawn to detect the hemorheological indexes, using an automatic analyzer 
TABLE 1: General Information of the two groups ( $X \pm$ S.E.M).

\begin{tabular}{|c|c|c|c|c|c|c|c|}
\hline \multirow{2}{*}{ Group } & \multirow{2}{*}{ Patients $(n)$} & \multicolumn{2}{|c|}{ Gender } & \multirow{2}{*}{ Age (years) } & \multirow{2}{*}{$\mathrm{CHD}(n)$} & \multirow{2}{*}{$\mathrm{CI}(n)$} & \multirow{2}{*}{$\mathrm{CHD}$ and $\mathrm{CI}(n)$} \\
\hline & & Male & Female & & & & \\
\hline Treatment Group & 60 & 34 & 26 & $76.6 \pm 7.81$ & 21 & 20 & 19 \\
\hline Control Group & 30 & 15 & 15 & $75.5 \pm 9.52$ & 13 & 14 & 3 \\
\hline Value & & \multicolumn{2}{|c|}{0.358} & $0.561^{*}$ & \multicolumn{3}{|c|}{5.157} \\
\hline$P$ & & \multicolumn{2}{|c|}{$>0.05$} & $>0.05$ & \multicolumn{3}{|c|}{$>0.05$} \\
\hline
\end{tabular}

Note: ${ }^{*}$ for $t$ value and the rest for $x^{2}$ value.

(Zhongchiweiye, ZL9000plus), including blood viscosity and redox viscosity at low (3/s) and high $(200 / \mathrm{s})$ shear rates, plasma viscosity at low shear rate (3/s), HCT, and erythrocyte aggregation index. The equipment was operated according to the protocols by the technicians at the Department of Clinical Laboratory in Shanghai Pudong District Cancer Prevention and Cure Hospital.

2.4. Blood Lipid Assays. Blood lipid profile was determined with Hitachi 7600 biochemistry automatic analyzer (Hitachi, Tokyo, Japan). Triglyceride (TG), total cholesterol (TC), and high-density lipoprotein cholesterol (HDLc) were measured with enzymatic methods. Low-density lipoprotein-cholesterol (LDL-c) was calculated according to the Friedewald formula. Apolipoprotein A-I (ApoA-I) and Apolipoprotein B (ApoB) were determined by immunoturbidimetric assays.

2.5. Statistical Analysis. Data are expressed as mean \pm S.E.M. Differences between means were tested for statistical significance by a $t$-test or $x^{2}$-test. The statistical level of significance was set at $P<0.05$.

\section{Results}

3.1. Changes of Blood Viscosity. As shown in Table 2, blood viscosity (high shear rate at $200 / \mathrm{s})(P<0.05)$, plasma viscosity (low shear rate at $3 / \mathrm{s})(P<0.05)$, and red blood cell aggregation $(P<0.01)$ decreased significantly after the therapy in the treatment group, but there were no significant changes in the control group. It was also shown that blood viscosity (low shear rate at $3 / \mathrm{s})$ kept unchanged $(P>0.05)$, and blood redox viscosity (high shear rate at 200/s or low shear rate at 3/s) decreased significantly $(P<0.01)$ after ILILT in the treatment group when the corresponding blood parameters became worse in the control treatment.

3.2. Changes of Blood Lipid Metabolite. As shown in Table 3, TC decreased significantly $(P<0.05)$ in the treatment group but there were no significant changes in the control group, LDL-c decreased significantly $(P<0.05)$ in the treatment group although LDL-c increased significantly $(P<0.001)$ in the control group, and HDL-c increased significantly $(P<0.01)$ from $1.07 \mathrm{mmol} / \mathrm{L}$ to $1.20 \mathrm{mmol} / \mathrm{L}$ but there were no significant changes in the control group. It was also shown that there were no changes in TG, ApoA-I, ApoB, and the ratio of ApoA-I to ApoB either in the treatment group or in the control group.

\section{Discussion}

The above results indicated that ILGLT improved blood lipid and hemorheologic behavior of patients with CHD or CI. These phenomena would be discussed from homeostatic viewpoint.

Homeostasis is one of the most remarkable and most typical properties of a highly complex open biosystem [13, 14]. It is a negative feedback response of a biosystem to maintain constant conditions inside the biosystem. This is a classic concept in physiology. However, oscillations are found at nearly every level of biology. It is too obscure to be studied so that it has been developed as functionspecific homeostasis (FSH) $[1,6]$. FSH is a negative-feedback response of a biosystem to maintain the function-specific fluctuations inside the biosystem so that the function is perfectly performed. A biosystem in a FSH means the function is in its FSH so that it is perfectly performed. A biosystem far from a FSH means the function is far from its FSH so that it is dysfunctional. A health person is in homeostasis so that all of his/her physiological functions are in their respective $\mathrm{FSH}$, and we have physiological functionspecific homeostasis (PhFSH) in which the physiological function can completely perform. A pathological function defined as a disease-specific physiological function which is far from the corresponding $\mathrm{PhFSH}$, but may be in its pathological function-specific homeostasis ( $\mathrm{PaFSH})$.

There was no direct PBM of LIL (LPBM) on a function in its FSH $[1,6]$. A function in its FSH can resist against weak disturbance such as LIL so that LIL cannot directly modulate a function in its FSH. Tables 2 and 3 suggested that the HCT, TG, Apo A-I, and Apo B of the patients with CHD or CI were in HCT-specific homeostasis (HSH), TG-specific homeostasis (TSH), Apo A-I-specific homeostasis (AaSH), and Apo Bspecific homeostasis (AbSH), respectively. Among these four kinds of FSH, the HSH, AaSH, and AbSH may be a kind of PhFSH $[15,16]$, but the TSH might be a kind of PaFSH [16].

There was LPBM on a function far from its FSH $[1,6]$, but the modulation mechanism of different functions may be different. One of the pathways mediating ILILT might be melatonin [7]. It has been found that ILILT can increase serum melatonin level $[6,7]$. Hoyos et al. have investigated the effect of melatonin, at pharmacological doses, on serum lipids of rats fed with a hypercholesterolemic 
TABLE 2: Blood viscosity index (mean \pm S.E.M).

\begin{tabular}{lcccccccc}
\hline \multirow{2}{*}{ BV } & \multicolumn{2}{c}{ Treatment group $(n)$} & \multicolumn{2}{c}{ Verified statistics } & \multicolumn{2}{c}{ Control group $(n)$} & \multicolumn{2}{c}{ Verified statistics } \\
& Before & After & $T$ & $P$ & Before & After & $T$ & $P$ \\
\hline BV(l) & $8.34 \pm 1.91$ & $8.15 \pm 1.91$ & 0.545 & $>0.05$ & $6.54 \pm 1.75$ & $7.87 \pm 1.99$ & 2.735 \\
BV(h) & $4.23 \pm 0.82$ & $3.94 \pm 0.74$ & 2.020 & $<0.05$ & $3.56 \pm 0.65$ & $4.02 \pm 1.64$ & 1.428 & $>0.05$ \\
PV & $1.23 \pm 0.02$ & $1.14 \pm 0.22$ & 2.233 & $<0.05$ & $1.23 \pm 0.18$ & $1.23 \pm 0.01$ & - & - \\
HCT & $39.88 \pm 7.72$ & $41.66 \pm 4.79$ & 1.526 & $>0.05$ & $37.92 \pm 6.04$ & $38.55 \pm 6.52$ & 0.388 & $>0.05$ \\
RV(l) & $17.35 \pm 4.03$ & $15.24 \pm 2.82$ & 3.323 & $<0.01$ & $14.09 \pm 3.26$ & $16.88 \pm 4.04$ & 2.944 & $<0.01$ \\
RV(h) & $6.99 \pm 0.75$ & $6.63 \pm 0.55$ & 2.120 & $<0.05$ & $6.27 \pm 1.17$ & $7.21 \pm 1.54$ & 2.662 & $<0.05$ \\
RBCA & $2.15 \pm 0.41$ & $1.95 \pm 0.24$ & 3.263 & $<0.01$ & $1.77 \pm 0.35$ & $1.92 \pm 0.23$ & 1.961 & $>0.05$ \\
\hline
\end{tabular}

BV: blood viscosity, WBV: Whole blood viscosity, PV: plasma viscosity at low shear rate (3/s), RV: redox viscosity, RBCA: red blood cell aggregation, HCT: hematocrit, h: high shear rate at 200/s, l: low shear rate at 3/s.

TABLE 3: Blood lipid index (mean \pm S.E.M).

\begin{tabular}{lcccccccc}
\hline \multirow{2}{*}{ BL } & \multicolumn{2}{c}{ Treatment group $(n)$} & \multicolumn{2}{c}{ Verified statistics } & \multicolumn{2}{c}{ Control group $(n)$} & \multicolumn{2}{c}{ Verified statistics } \\
& Before & After & $T$ & $P$ & Before & After & $T$ & $P$ \\
\hline TC $(\mathrm{mmol} / \mathrm{L})$ & $4.44 \pm 1.72$ & $3.78 \pm 0.95$ & 2.603 & $<0.05$ & $4.10 \pm 1.15$ & $4.07 \pm 1.26$ & 0.096 & $>0.05$ \\
TGs $(\mathrm{mmol} / \mathrm{L})$ & $1.88 \pm 0.67$ & $1.70 \pm 0.77$ & 1.366 & $>0.05$ & $1.85 \pm 0.71$ & $1.87 \pm 0.70$ & 0.110 & $>0.05$ \\
HDL-c(mmol/L) & $1.07 \pm 0.27$ & $1.20 \pm 0.24$ & 2.790 & $<0.01$ & $1.00 \pm 0.31$ & $1.17 \pm 0.41$ & 1.812 & $>0.05$ \\
LDL-c $(\mathrm{mmol} / \mathrm{L})$ & $2.73 \pm 0.76$ & $2.48 \pm 0.51$ & 2.115 & $<0.05$ & $1.39 \pm 0.70$ & $2.33 \pm 0.93$ & 4.424 & $<0.001$ \\
ApoA-I (g/L) & $1.29 \pm 0.29$ & $1.35 \pm 0.27$ & 1.367 & $>0.05$ & $1.24 \pm 0.26$ & $1.22 \pm 0.34$ & 0.256 & $>0.05$ \\
ApoB $(\mathrm{g} / \mathrm{L})$ & $0.89 \pm 0.29$ & $0.81 \pm 0.19$ & 1.788 & $>0.05$ & $0.81 \pm 0.29$ & $0.86 \pm 0.33$ & 0.125 & $>0.05$ \\
A/B & $1.58 \pm 0.43$ & $1.64 \pm 0.42$ & 0.733 & $>0.05$ & $1.54 \pm 0.44$ & $1.55 \pm 0.49$ & 0.083 & $>0.05$ \\
\hline
\end{tabular}

BL: blood lipid, TC: total cholesterol, TGs: triglycerides, HDL-c(LDL-c): high(low)-density lipoprotein cholesterol, ApoA-I(ApoB): Apolipoprotein AI(Apolipoprotein B), A/B: ApoA-I/ApoB.

diet [17]. Different groups of animals were fed with either the regular Sanders Chow diet or a diet enriched in cholesterol. Moreover, animals were treated with or without melatonin in the drinking water for 3 months. They found that melatonin treatment did not affect the levels of cholesterol or triglycerides in rats fed with a regular diet. However, the increase in TC and LDL-c induced by a cholesterol-enriched diet was reduced significantly by melatonin administration. On the other hand, melatonin administration prevented the decrease in HDL-c induced by the same diet. However, no differences in the levels of TGs were found. Obviously, ILGLT and melatonin play roles similar to each other on TGs, HDLc, and LDL-c as Table 3 has shown.

Nicotinamide adenine dinucleotide $\left(\mathrm{NAD}^{+}\right)$might also be one of the pathways mediating ILILT [7]. ILILT can increase serum melatonin level $[6,7]$ and then $\mathrm{NAD}^{+}$level [18]. ILILT might directly increase the ratio of intracellular $\mathrm{NAD}^{+}$level and the intracellular level of its reduced form $\mathrm{NADH}, \mathrm{NAD}^{+} / \mathrm{NADH}$. Karu has studied the cellular response of LIL from the viewpoint of cellular redox potential and suggested that the cellular response is absent when the redox potential is optimal, and stronger when the redox potential of the target cell is initially shifted to a more reduced state [19]. The cellular redox potential might be represented by $\mathrm{NAD}^{+} / \mathrm{NADH}$ [19]. A normally functioning cell in its FSH has its specific redox potential and $\mathrm{NAD}^{+} / \mathrm{NADH}$, respectively, which are referred as the FSH-specific redox potential (FSR) and the FSH-specific $\mathrm{NAD}^{+} / \mathrm{NADH}(\mathrm{FSN})$, respectively. A dysfunctional cell far from its FSH is initially shifted to a more reduced state with lowered $\mathrm{NAD}^{+} / \mathrm{NADH}$. In terms of Karu's suggestion [19], the magnitude of LPBM is determined by redox potential or $\mathrm{NAD}^{+} / \mathrm{NADH}$ of the cell at the moment. LPBM may enhance the redox potential and $\mathrm{NAD}^{+} / \mathrm{NADH}$, respectively, in cells far from their FSH. The lower the redox potential below the FSR is, the lower the $\mathrm{NAD}^{+} / \mathrm{NADH}$ below FSN will be, and the stronger the LPBM will finally be in terms of Karu's suggestion [19]. There is connexin 43 (Cx43), a NAD ${ }^{+}$transporter, in the cellular membrane so that there is bidirectional $\mathrm{NAD}^{+}$transport across cellular membrane [20]. ILILT may enhance $\mathrm{NAD}^{+}$level in the irradiated blood cells, and then in the blood through $\mathrm{Cx} 43$, and then in the unirradiated cells through $\mathrm{Cx} 43$, which increase $\mathrm{NAD}^{+} / \mathrm{NADH}$ in both the irradiated cells and the un-irradiated cells. Niacin is a precursor of $\mathrm{NAD}^{+}$and, as such, may increase $\mathrm{NAD}^{+} / \mathrm{NADH}$ [21]. Montefusco et al. have investigated whether acipimox, a nicotinic acid analog, improved hemorheological parameters [22]. 21 patients (17 Males, 4 Females) with asymptomatic hypertriglyceridemia were treated with acipimox (250 b.i.d.) for 30 days. They found that blood viscosity decreased $(P<$ 0.05 and $<0.01$ ) (range of reduction 6-20\%) at all shear rates examined (from $2.25 \mathrm{~s}^{-1}$ to $450 \mathrm{~s}^{-1}$ ) and plasma viscosity was significantly reduced only at lower shear rates (2.25 and $4.50 \mathrm{~s}^{-1}$ ). In type 2 hypertensive diabetes patients, niceritrol, a pentaerythritol ester containing four niacin residues and aspirin (162/mg per day) that reduced blood viscosity at low $\left(37.6 \mathrm{~s}^{-1}\right)$ and high $\left(376 \mathrm{~s}^{-1}\right)$ shear rates, did not modulate HCT [23]. Obviously, ILGLT and niacin played roles similar 
to each other on blood and plasma viscosity as Table 2 has shown.

\section{Conclusion}

ILGLT may improve blood lipid and hemorheologic behavior of patients with CHD or CI.

\section{Conflict of Interests}

No competing financial interests exist.

\section{Acknowledgments}

This work was supported by National Science Foundation of China (60878061), and the Opening Project of MOE Key laboratory of Laser Life Science, South China Normal University, Guangzhou, China.

\section{References}

[1] T. C. Y. Liu, R. Liu, L. Zhu, J. Q. Yuan, M. Hu, and S. H. Liu, "Homeostatic photobiomodulation," Frontiers of Optoelectronics in China, vol. 2, no. 1, pp. 1-8, 2009.

[2] L. Baratto, L. Calzà, R. Capra et al., "Ultra-low-level laser therapy," Lasers in Medical Science, vol. 26, no. 1, pp. 103-112, 2011.

[3] R. K. Tulebaev, S. B. Sadykov, V. A. Romanov, and G. K. Khalitova, "Indicators of the activity of the immune system during laser therapy of vasomotor rhinitis," Vestnik Otorinolaringologii, no. 1, pp. 46-49, 1989 (Russian).

[4] B. V. Shevrygin, S. V. Rybalkin, F. F. Pekli, and L. V. Feniksova, "Correction of microcirculatory disorders with low-energy laser radiation in children with vasomotor rhinitis," Vestnik Otorinolaringologii, no. 2, pp. 31-33, 2000 (Russian).

[5] I. Kruchinina, L. V. Feniksova, S. V. Rybalkin, and F. F. Pekli, "Therapeutic effect of helium-neon laser on microcirculation of nasal mucosa in children with acute and chronic maxillary sinusitis as measured by conjunctival biomicroscopy," Vestnik Otorinolaringologii, no. 3, pp. 26-30, 1991 (Russian).

[6] C. Liu and P. Zhu, Intranasal Low Intensity Laser Therapy, People's Military Medical Press, Beijing, China, 2009.

[7] T. C. Y. Liu, D. F. Wu, Z. Q. Gu, and M. Wu, "Applications of intranasal low intensity laser therapy in sports medicine," Journal of Innovation in Optical Health Science, vol. 3, no. 1, pp. 1-16, 2010.

[8] S. Gao, Nose Therapy, Huaxia Publishing House, Beijing, China, 1994.

[9] M. L. Noback, K. Harvati, and F. Spoor, "Climate-related variation of the human nasal cavity," American Journal of Physical Anthropology, vol. 145, no. 4, pp. 599-614, 2011.

[10] R. S. Wilson, L. Yu, and D. A. Bennett, "Odor identification and mortality in old age," Chemical Senses, vol. 36, no. 1, pp. 63-67, 2011.

[11] H. Tuby, L. Maltz, and U. Oron, "Induction of autologous mesenchymal stem cells in the bone marrow by low-level laser therapy has profound beneficial effects on the infarcted rat heart," Lasers in Surgery and Medicine, vol. 43, no. 5, pp. 401409, 2011.

[12] J. H. Ko, T. B. J. Kuo, and G. S. Lee, "Effect of postural change on nasal airway and autonomic nervous system established by rhinomanometry and heart rate variability analysis," American Journal of Rhinology, vol. 22, no. 2, pp. 159-165, 2008.

[13] W. B. Cannon, The Wisdom of the Body, W. W. Norton, New York, NY, USA, 1932.

[14] D. S. Jones, Textbook of Functional Medicine, The Institute for Functional Medicine, Gig Harbor, Wash, USA, 2006.

[15] T. Kunnas, T. Solakivi, K. Huuskonen, A. Kalela, J. Renko, and S. T. Nikkari, "Hematocrit and the risk of coronary heart disease mortality in the TAMRISK study, a 28-year follow-up," Preventive Medicine, vol. 49, no. 1, pp. 45-47, 2009.

[16] G. Walldius and I. Jungner, "Apolipoprotein B and apolipoprotein A-I: risk indicators of coronary heart disease and targets for lipid-modifying therapy," Journal of Internal Medicine, vol. 255, no. 2, pp. 188-205, 2004.

[17] M. Hoyos, J. M. Guerrero, R. Perez-Cano et al., "Serum cholesterol and lipid peroxidation are decreased by melatonin in diet-induced hypercholesterolemic rats," Journal of Pineal Research, vol. 28, no. 3, pp. 150-155, 2000.

[18] M. Bubis and N. Zisapel, "A role for $\mathrm{NAD}^{+}$and cADP-ribose in melatonin signal transduction," Molecular and Cellular Endocrinology, vol. 137, no. 1, pp. 59-67, 1998.

[19] T. Karu, The Science of Low-Power Laser Therapy, Gordon and Breach Science Publishers, Amsterdam, The Netherlands, 1998.

[20] S. Bruzzone, L. Guida, E. Zocchi, L. Franco, and A. De Flora, "Connexin 43 hemi channels mediate $\mathrm{Ca}^{2+}$-regulated transmembrane $\mathrm{NAD}^{+}$fluxes in intact cells," FASEB Journal, vol. 15, no. 1, pp. 10-12, 2001.

[21] R. S. Rosenson, "Antiatherothrombotic effects of nicotinic acid," Atherosclerosis, vol. 171, no. 1, pp. 87-96, 2003.

[22] S. Montefusco, G. Iannaci, A. Gnasso, C. Cortese, F. Lamenza, and A. Postiglione, "Blood and plasma viscosity after acipimox treatment in hypertriglyceridemic patients," International Journal of Clinical Pharmacology Therapy and Toxicology, vol. 26, no. 10, pp. 492-494, 1988.

[23] T. Hamazaki, K. Hasunuma, and S. Kobayashi, "The effects of lipids, blood viscosity and platelet aggregation of combined use of niceritrol (Perycit) and a low dose of acetylsalicylic acid," Atherosclerosis, vol. 55, no. 1, pp. 107-113, 1985. 


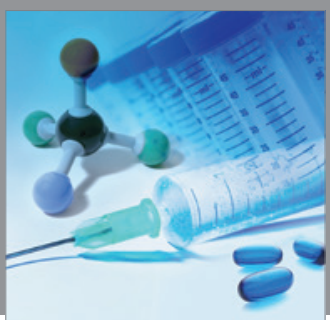

International Journal of

Medicinal Chemistry

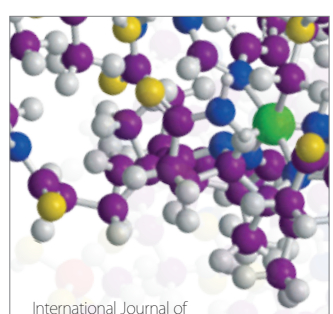

Carbohydrate Chemistry

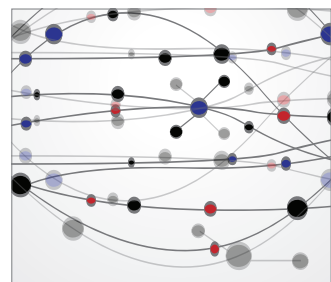

The Scientific World Journal
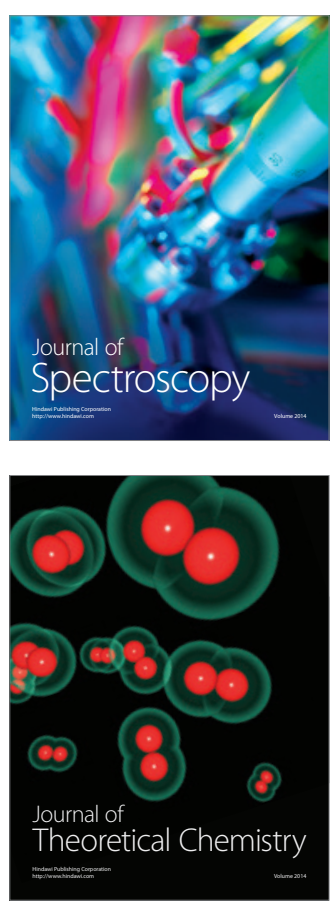
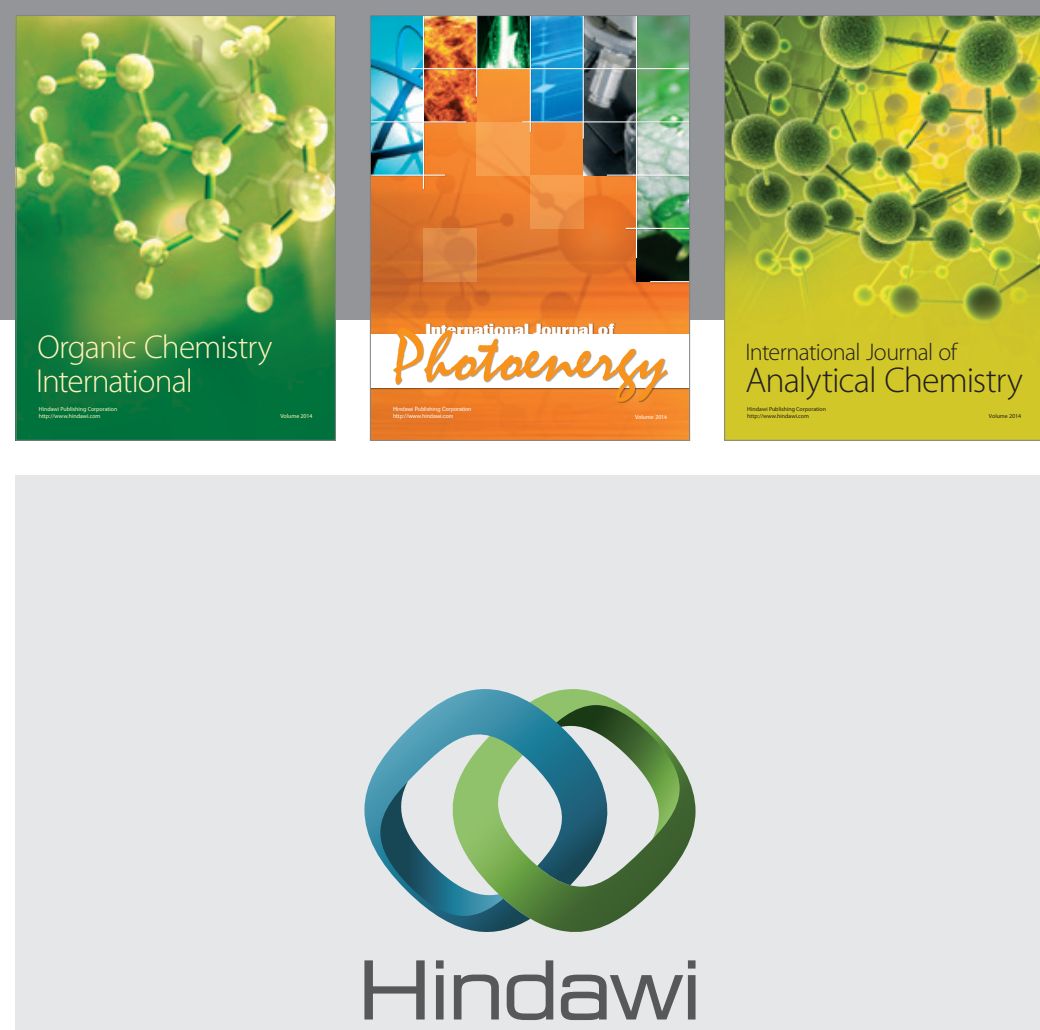

Submit your manuscripts at

http://www.hindawi.com
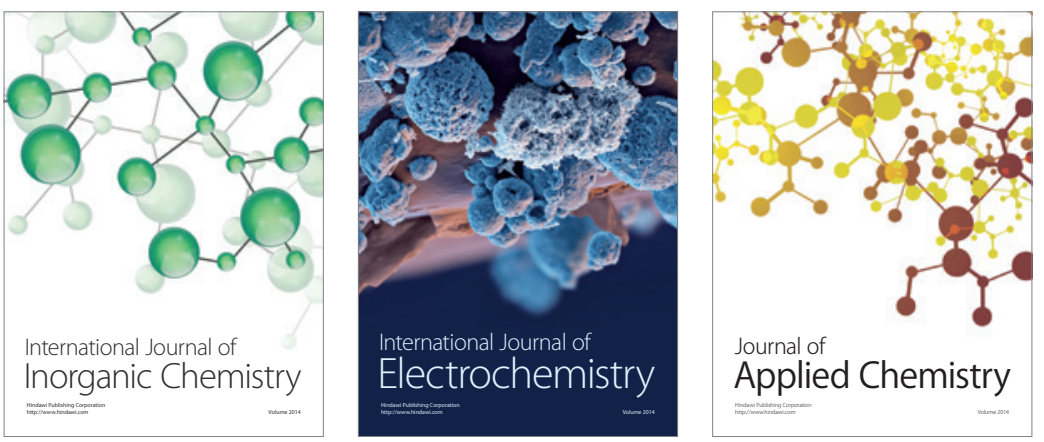

Journal of

Applied Chemistry
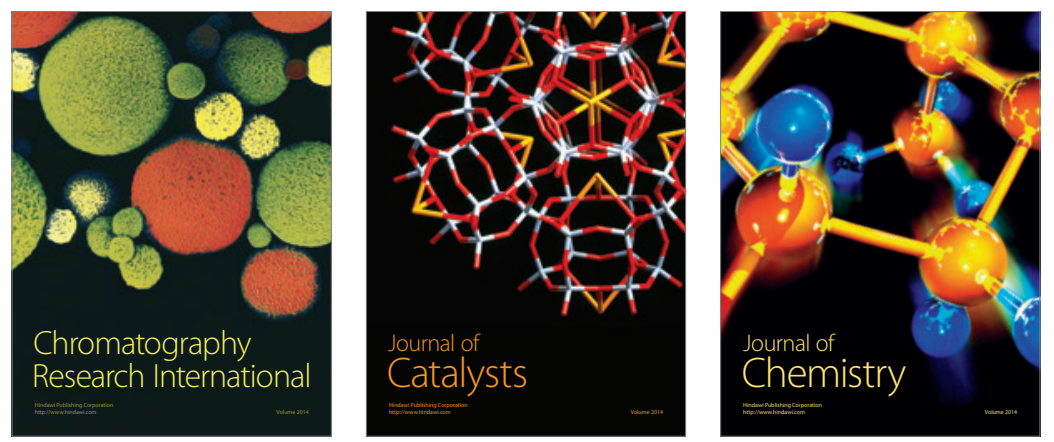
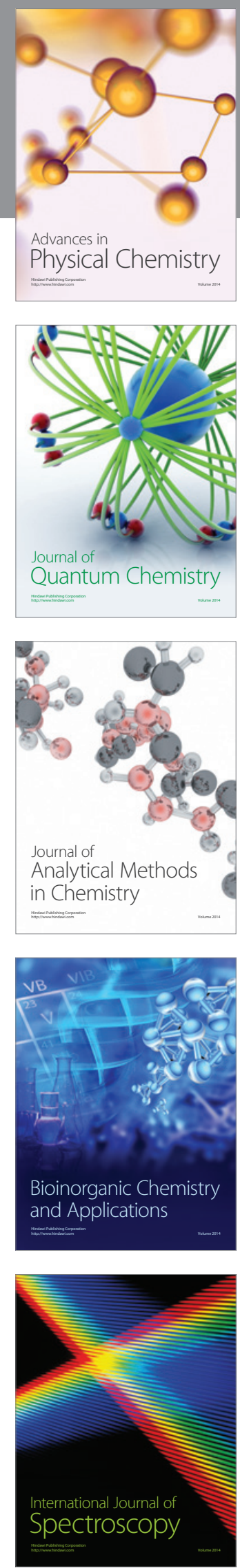